\title{
Prognostic Role of Docetaxel-induced Suppression of Free Testosterone Serum Levels in Metastatic Prostate Cancer Patients
}

\author{
Paula Kappler \\ Hannover Medical School \\ Stefan Brunotte \\ Hannover Medical School \\ Philipp Ivanyi \\ Hannover Medical School \\ Michael Morgan \\ Hannover Medical School

\section{Arnold Ganser} \\ Hannover Medical School \\ Christoph Reuter ( $\nabla$ reuter.christoph@mh-hannover.de) \\ Hannover Medical School
}

\section{Research Article}

Keywords: prostate cancer, free testosterone, docetaxel

Posted Date: May 6th, 2021

DOI: https://doi.org/10.21203/rs.3.rs-464459/v1

License: (9) (i) This work is licensed under a Creative Commons Attribution 4.0 International License. Read Full License

Version of Record: A version of this preprint was published at Scientific Reports on August 12th, 2021. See the published version at https://doi.org/10.1038/s41598-021-95874-y. 


\section{Abstract}

To date, only few data concerning the biologically active, free form of testosterone (FT) are available in metastatic prostate cancer ( $\mathrm{mPC}$ ) and its impact on disease, therapy and outcome is largely unknown. We retrospectively studied the effect of docetaxel on FT and total testosterone (TT) serum levels in 67 mPC patients between April 2008 and November 2020. FT and TT levels were measured before and weekly during therapy. The primary endpoint was overall survival (OS). Secondary endpoints were prostate-specific antigen and radiographic response (PSAR, RR), progression-free survival (PFS), FT/TT levels and safety. Median FT and TT serum levels were completely suppressed below the detection limit during docetaxel treatment (FT: from $0.32 \mathrm{pg} / \mathrm{mL}$ to $<0.18 \mathrm{pg} / \mathrm{mL}$ and TT: from 0.12 to $<0.05 \mathrm{ng} / \mathrm{mL}$, respectively). Multivariate Cox regression analyses identified requirement of non-narcotics, PSAR, complete FT suppression and FT nadir values $<0.18 \mathrm{pg} / \mathrm{mL}$ as independent parameters for PFS. Prior androgen-receptor targeted therapy (ART), soft tissue metastasis and complete FT suppression were independent prognostic factors for OS. FT was not predictive for treatment outcome in MPC patients with a history of ART.

\section{Introduction}

Prostate cancer (PC) growth and progression is androgen-dependent and androgen deprivation therapy (ADT) is an effective strategy to manage advanced disease [1-3]. Historically, suppression of total testosterone (TT) to a level lower than $50 \mathrm{ng} / \mathrm{dL}(<1.7 \mathrm{nmol} / \mathrm{L})$ has been defined as castration, however, a testosterone suppression target of less than $20 \mathrm{ng} / \mathrm{dL}$ improves patient survival and delays tumor progression [1, 4]. Androgen receptor (AR) targeted therapy (ART) in combination with ADT leads to testosterone suppression to near zero and further improves patient survival [1]. Castration-resistance occurs despite castrate testosterone levels through reactivation of AR pathways from multiple mechanisms. Furthermore, neuroendocrine transdifferentation may also occur in PC and lead to castration resistance. Shorter progression-free (PFS) and overall survival (OS) are the results [1]. Due to its critical role, testosterone levels should be monitored frequently during therapy [1].

Ryan et al. stated that docetaxel therapy significantly reduces androgen levels including total testosterone (TT), with increased OS (26.3 vs. 20.9 months) favoring patients with high versus low decline, respectively [5]. Total serum androgens (TT, androstenedione, DHEAS) were described as important biomarkers in PC treatment and may be useful in risk stratification in future studies $[5,6]$.

Plasma testosterone (total testosterone, TT) circulates specifically bound to sex hormone-binding globulin (SHBG) (50-70\%), nonspecifically bound to albumin (20-30\%), other proteins (4\%) and unbound $(1-3 \%)$, or free (FT) [7]. Bioavailable testosterone (BT) is FT plus albumin-bound testosterone [7, 8]. A method to efficiently evaluate BT is the measurement of FT [8]. Although a key target in the treatment of advanced prostate cancer, the role of FT to PC treatment and outcome are largely unknown [9]. The castration level of FT was established at $<1.7 \mathrm{pg} / \mathrm{mL}(<5.9 \mathrm{pmol} / \mathrm{L})$ [8]. Von Klot et Al. identified FT $<0.5$ 
$\mathrm{pg} / \mathrm{mL}$ as a prognostic marker in second-line therapy, which is associated with improved cancer-related survival (43.6 vs. 17.3 months) [10].

Docetaxel seems to be less efficient after treatment with ART, with a reduced PSAR rate after abiraterone (26\%) and significantly shorter PFS and OS after enzalutamide. A certain cross-resistance of docetaxel and ART was discussed $[11,12]$. Studies suggest that docetaxel exerts an effect on androgen receptor signaling, while testosterone impairs cellular uptake of docetaxel and inhibits stabilization of microtubules, leading to the proposal that testosterone levels should be decreased during chemotherapy with docetaxel $[13,14]$.

Even in the castration-resistant setting, PC tumors still rely on androgen signaling [15]. Hence, the goal of this study was to investigate the effect of docetaxel on FT and TT in different stages of metastatic PC (e.g. castration-naïve (mCNPC, group 1), castration-resistant (mCRPC, group 2) and castration-resistant patients with prior ART (mCRPC-ART, group 3)).

\section{Methods}

\section{Patients}

For this translational biomarker study, data from patients with $\mathrm{mPC}$ treated with docetaxel at Hannover Medical School from April 2008-November 2020 were retrospectively analyzed and followed up until death or until December 2020. Eligibility criteria were a histologically confirmed mPC and disease progression according to the PCWG $2 / 3$ criteria $[4,16]$. Patients who received at least two cycles of docetaxel were included. All data were collected following patient informed consent, in accordance with the principles of the Declaration of Helsinki and Good Clinical Practice guidelines, and with the Hanover Medical School institutional review board approval (13. August 2008). Patients were stratified into three subgroups: (1) castration-naïve disease (mCNPC), (2) castration-resistant disease (mCRPC) and (3) mCRPC patients with a history of ART (mCRPC-ART).

\section{Treatment plan}

At baseline, medical history and physical examination were performed, including an initial staging. Patients received $75 \mathrm{mg} / \mathrm{m}^{2}$ of docetaxel intravenously every three weeks (q3w), $50 \mathrm{mg} / \mathrm{m}^{2}$ every two weeks (q2w) or 30-35 mg/m² weekly on days 1, 8 and 15 (q1w). Concomitant use of dexamethasone and oral prednisone $(5 \mathrm{mg})$ twice a day were part of the regimen. ADT was continued throughout therapy. Morning FT and TT (8-11 am) were obtained before and weekly during treatment (on average 28 samples per patient) using an enzyme immunoassay (ELISA from IBL, International GmbH, Hamburg, Germany) and a direct, competitive, chemiluminescence immunoassay (CLIA) (LIAISON® Testosterone Assay, Diasorin S.p.A., Saluggia, Italy). PSA levels, CEA levels, neuroendocrine tumor markers (neuronspecific enolase (NSE), chromogranin A ( $\mathrm{CgA})$ ), lactate dehydrogenase (LDH), alkaline phosphatase (AP), hemoglobin and analgesic requirement were parameters at baseline to possibly predict OS [16]. Patients 
were assessed according to PCWG2/3 criteria and RECIST 1.1 by CT and bone scan every three months or if tumor progression was suspected $[4,17,18]$.

\section{Data analysis}

The primary study end point was OS, defined by the initiation of docetaxel therapy until death. Secondary endpoints were PFS, defined as the time between the start of docetaxel therapy until progression according to PCWG $2 / 3$ criteria $[4,16]$, PSA response (PSAR), which is defined by a decline of $>50 \%$ from baseline, and FT reduction of $100 \%$ from baseline and safety. Radiographic response (RR) was evaluated according to RECIST 1.1. [17]. Follow-up data were collected throughout December 2020.

Statistical analyses were performed using SPSS statistics v26.0. Categorical variables were summarized, numeric variables were analyzed in median and range. Logistic regression was used to estimate the prognostic significance of FT suppression in predicting $\geq 50 \%$ decline in PSA from baseline. To determine the prognostic significance of baseline characteristics on PFS and OS, Cox proportional hazards regression modeling was used. Chi-square tests and t-tests were applied to estimate $p$ values of variables at baseline. Uni-and multivariate Cox regression analyses were used to demonstrate the impact of covariates on PFS and OS. Non-proportionality was assessed by plotting the Kaplan-Meier survival distribution as a function of the survival time for each level of the covariate and plotting the function log(log(survival probability)) as a function of the log survival time [19]. Additionally, extended Cox modelling with time-by-covariates and conditional landmark analyses were used to remove potential guarantee-time bias, specifically the time-window bias, which is introduced because of differential exposure opportunity time windows between subjects [20]. Only $p$-values of $<0.05$ were considered statistically significant for all comparisons.

\section{Results}

\section{Patient characteristics}

A total of 67 patients with a histologically confirmed metastatic adenocarcinoma of the prostate were analyzed. Seven patients were castration-naïve (mCNPC, group 1), 26 patients were castration-resistant after ADT (mCRPC, group 2) and 34 patients were castration resistant after ADT plus ART (mCRPC-ART, group 3). The median age at time of diagnosis was 69 years (Table 1 ). 
Table 1

- Patient characteristics, prior treatments and laboratory values at baseline before start of docetaxel chemotherapy for 67 patients.

\begin{tabular}{|c|c|c|c|c|c|}
\hline & $\begin{array}{l}\text { All } \\
n=67\end{array}$ & $\begin{array}{l}\text { Group } \\
1 \\
\text { mCNPC } \\
n=7\end{array}$ & $\begin{array}{l}\text { Group } 2 \\
\text { mCRPC } \\
n=26\end{array}$ & $\begin{array}{l}\text { Group } 3 \\
\text { mCRPC-ART } \\
n=34\end{array}$ & $p$-value \\
\hline Age, median & 69 & 69 & 68.5 & 69 & \\
\hline ECOG, no.(\%) & $22(32.8)$ & $3(42.9)$ & 12 & $7(20.6)$ & 0.009 \\
\hline 0 & $31(46.3)$ & $4(57.1)$ & & $16(47.1)$ & \\
\hline 1 & 10 (14.9) & 0 & $(42.3)$ & $7(20.6)$ & \\
\hline 2 & $4(6)$ & 0 & $3(11.5)$ & $4(11.8)$ & \\
\hline 3 & & & 0 & & \\
\hline Gleason score $8-10$, no(\%) & $36(53.7)$ & $2(28.6)$ & $\begin{array}{l}15 \\
(57.7)\end{array}$ & $19(55.9)$ & \\
\hline BMI & 26.2 & 24.7 & 27.8 & 25.3 & 0.025 \\
\hline Metastases & $28(41.8)$ & $2(28.6)$ & $13(50)$ & $13(38.2)$ & \\
\hline No. of organs (\%) & $24(35.8)$ & $3(42.8)$ & 5 (19.2) & $16(47.1)$ & \\
\hline 1 & $15(22.4)$ & $2(28.6)$ & $8(30.8)$ & $5(14.7)$ & \\
\hline \multicolumn{6}{|l|}{2} \\
\hline \multicolumn{6}{|l|}{$\geq 3$} \\
\hline Bone & $64(95.5)$ & $7(100)$ & \multirow{2}{*}{$\begin{array}{l}25 \\
(96.2)\end{array}$} & $32(94.1)$ & \\
\hline Soft tissue & $42(62.7)$ & 5 (71.4) & & $23(67.7)$ & \\
\hline Lymph nodes & $34(50.8)$ & $5(71.4)$ & $\begin{array}{l}14 \\
(53.9)\end{array}$ & $17(50)$ & \\
\hline Lungs & $11(16.4)$ & $1(14.3)$ & $\begin{array}{l}12 \\
(46.2)\end{array}$ & $4(11.8)$ & \\
\hline Liver & $11(16.4)$ & 0 & \multirow{2}{*}{$\begin{array}{l}6(23.1) \\
5(19.2)\end{array}$} & $6(17.7)$ & \\
\hline \multirow[t]{2}{*}{ Brain } & \multirow[t]{2}{*}{$2(3)$} & 0 & & 0 & \\
\hline & & & $2(7.7)$ & & \\
\hline
\end{tabular}

ECOG = Eastern cooperative oncology group; $\mathrm{BMI}=$ body mass index; TUR-prostate $=$ transurethral resection of the prostate; $R T X$ = radiation therapy; PSMA = prostate-specifc membrane antigen; $A D T=$ androgen-depleting therapy; ART = androgen receptor targeted therapy; PSA = prostate- specific antigen; $\mathrm{FT}=$ free testosterone; $\mathrm{TT}=$ total testosterone; $\mathrm{Hb}=$ Hemoglobin; $\mathrm{AP}=$ alkaline phosphatase; $\mathrm{ULN}=$ upper limit of normal; $\mathrm{LDH}=$ lactate dehydrogenase; NSE = neuron-specific enolase; $\mathrm{CgA}=$ chromogranin A; NLR = neutrophil to lymphocyte ratio. 


\begin{tabular}{|c|c|c|c|c|c|}
\hline & $\begin{array}{l}\text { All } \\
n=67\end{array}$ & $\begin{array}{l}\text { Group } \\
1 \\
\text { mCNPC } \\
n=7\end{array}$ & $\begin{array}{l}\text { Group } 2 \\
\text { mCRPC } \\
n=26\end{array}$ & $\begin{array}{l}\text { Group } 3 \\
\text { mCRPC-ART } \\
n=34\end{array}$ & $p$-value \\
\hline $\begin{array}{l}\text { Cancer pain, no.(\%) } \\
\text { Non-narcotics required } \\
\text { Narcotics required }\end{array}$ & $\begin{array}{l}38(56.7) \\
26(38.8) \\
23(34.3)\end{array}$ & $\begin{array}{l}6(85.7) \\
4(57.1) \\
2(28.6)\end{array}$ & $\begin{array}{l}14 \\
(53.9) \\
8(30.8) \\
7(26.9)\end{array}$ & $\begin{array}{l}18(52.9) \\
14(41.2) \\
14(41.2)\end{array}$ & \\
\hline $\begin{array}{l}\text { Prior treatment } \\
\text { Local therapy } \\
\text { Radical prostactetomy, no. } \\
(\%) \\
\text { TUR-prostate, no.(\%) }\end{array}$ & $\begin{array}{l}24(35.8) \\
13(19.4)\end{array}$ & $\begin{array}{l}0 \\
0\end{array}$ & $\begin{array}{l}13(50) \\
7(26.9)\end{array}$ & $\begin{array}{l}11(32.4) \\
6(17.7)\end{array}$ & 0.015 \\
\hline $\begin{array}{l}\text { Radiotherapy } \\
\text { RTX prostate, no.(\%) } \\
\text { RTX bone, no.(\%) } \\
\text { RTX soft tissue, no.(\%) }\end{array}$ & $\begin{array}{l}19(28.4) \\
34(50.8) \\
10(14.9)\end{array}$ & $\begin{array}{l}0 \\
3(42.9) \\
0\end{array}$ & $\begin{array}{l}8(30.8) \\
14 \\
(53.9) \\
4(15.4)\end{array}$ & $\begin{array}{l}11(32.4) \\
17(50) \\
6(17.7)\end{array}$ & \\
\hline $\begin{array}{l}\text { Radiopharmaceuticals } \\
\text { Alpharadin, no.(\%) } \\
\text { PSMA ligands, no.(\%) }\end{array}$ & $\begin{array}{l}3(4.5) \\
1(1.5)\end{array}$ & $\begin{array}{l}0 \\
0\end{array}$ & $\begin{array}{l}0 \\
0\end{array}$ & $\begin{array}{l}3(8.8) \\
1(2.9)\end{array}$ & \\
\hline Duration of $A D T$ in months & $17(0-108)$ & 0 & 22.5 & 25 & 0.008 \\
\hline ART & $29(43.3)$ & 0 & 0 & $29(85.3)$ & $<0.001$ \\
\hline Abiraterone, no.(\%) & $20(29.9)$ & 0 & 0 & $20(58.8)$ & $<0.001$ \\
\hline Enzalutamide, no.(\%) & & & & & 0.009 \\
\hline Ketoconazole, no.(\%) & $2(3)$ & 0 & $1(3.9)$ & $1(2.9)$ & \\
\hline Estramustine, no.(\%) & $5(7.5)$ & 0 & $4(15.4)$ & $1(2.9)$ & \\
\hline Prior docetaxel, no.(\%) & $18(26.8)$ & 0 & $5(19.2)$ & $13(38.2)$ & \\
\hline
\end{tabular}

ECOG = Eastern cooperative oncology group; $\mathrm{BMI}=$ body mass index; TUR-prostate = transurethral resection of the prostate; RTX = radiation therapy; PSMA = prostate-specifc membrane antigen; $A D T=$ androgen-depleting therapy; ART = androgen receptor targeted therapy; PSA = prostate- specific antigen; $\mathrm{FT}=$ free testosterone; $\mathrm{TT}=$ total testosterone; $\mathrm{Hb}=$ Hemoglobin; $\mathrm{AP}=$ alkaline phosphatase; $\mathrm{ULN}=$ upper limit of normal; $\mathrm{LDH}=$ lactate dehydrogenase; NSE = neuron-specific enolase; $\mathrm{CgA}=$ chromogranin A; NLR = neutrophil to lymphocyte ratio. 


\begin{tabular}{|c|c|c|c|c|c|}
\hline & $\begin{array}{l}\text { All } \\
n=67\end{array}$ & $\begin{array}{l}\text { Group } \\
1 \\
\text { mCNPC } \\
n=7\end{array}$ & $\begin{array}{l}\text { Group } 2 \\
\text { mCRPC } \\
n=26\end{array}$ & $\begin{array}{l}\text { Group } 3 \\
\text { mCRPC-ART } \\
n=34\end{array}$ & $p$-value \\
\hline $\begin{array}{l}\text { Laboratory at baseline } \\
\text { PSA ( } \mu \mathrm{g} / \mathrm{L}) \text {, median (range) }\end{array}$ & $\begin{array}{l}129.7(4.26- \\
6695)\end{array}$ & 737.9 & 102.85 & 128.3 & 0.001 \\
\hline FT (pg/mL), median (range) & $0.315(0.18-15.3)$ & 1.92 & 1.14 & 0.18 & $<0.001$ \\
\hline TT (ng/mL), median (range) & $0.12(0.05-4.86)$ & 2.4 & 0.19 & 0.12 & $<0.001$ \\
\hline $\mathrm{Hb}(\mathrm{g} / \mathrm{dL})$, median (range) & $11.5(7.4-15.6)$ & 10.2 & 12.1 & 11.5 & 0.029 \\
\hline AP (U/L), median (range) & $151(44-4834)$ & 307 & 122 & 146 & \\
\hline $\begin{array}{l}>\text { ULN, no. }(\%) \\
>2 x U L N, \text { no. }(\%)\end{array}$ & $\begin{array}{l}36(53.7) \\
23(34.3)\end{array}$ & $\begin{array}{l}7(100) \\
5(71.4)\end{array}$ & $\begin{array}{l}12 \\
(46.2) \\
7(26.9)\end{array}$ & $\begin{array}{l}17(50) \\
11(32.4)\end{array}$ & \\
\hline LDH (U/L), median (range) & $285(158-1266)$ & 422 & 278 & 276.5 & \\
\hline $\begin{array}{l}>\text { ULN, no. }(\%) \\
>2 x \text { ULN, no. }(\%)\end{array}$ & $41(61.2)$ & $\begin{array}{l}6(85.7) \\
1(14.3)\end{array}$ & $\begin{array}{l}16 \\
(61.5) \\
6(23.1)\end{array}$ & $\begin{array}{l}19(55.9) \\
6(17.7)\end{array}$ & \\
\hline $\begin{array}{l}\text { NSE }(\mu \mathrm{g} / \mathrm{L}), \text { median (range) } \\
>\mathrm{ULN}, \mathrm{no} .(\%)\end{array}$ & $\begin{array}{l}21(11-56) \\
40(59.7)\end{array}$ & $\begin{array}{l}22.5 \\
5(71.4)\end{array}$ & $\begin{array}{l}20 \\
13(50)\end{array}$ & $\begin{array}{l}23 \\
22(64.7)\end{array}$ & \\
\hline $\mathrm{CgA}(\mu \mathrm{g} / \mathrm{L})$, median (range) & $149.5(2-664)$ & 177 & 90 & 189 & 0.018 \\
\hline >ULN, no.(\%) & $42(62.7)$ & $6(85.7)$ & $13(50)$ & $23(67.7)$ & \\
\hline CgA/NSE > ULN, no.(\%) & $49(73.1)$ & $7(100)$ & \multirow{2}{*}{$\begin{array}{l}17 \\
(65.4) \\
9(34.6)\end{array}$} & $26(76.5)$ & \\
\hline CgA and NSE > ULN, no(\%) & $31(61.2)$ & $4(57.1)$ & & $19(55.9)$ & \\
\hline NLR, median & 5.8 & 5.3 & 4.9 & 6.9 & \\
\hline $\begin{array}{l}\text { ECOG = Eastern cooperative o } \\
\text { resection of the prostate; RTX } \\
\text { androgen-depleting therapy; } A \\
\text { antigen; } F T=\text { free testosteron } \\
\text { ULN = upper limit of normal; } L \\
\text { chromogranin A; NLR = neutro }\end{array}$ & $\begin{array}{l}\text { ology group; BMI = b } \\
\text { adiation therapy; PS } \\
=\text { androgen recepto } \\
\text { T = total testosteror } \\
\text { = lactate dehydrog } \\
\text { il to lymphocyte rat }\end{array}$ & $\begin{array}{l}y \text { mass } \\
\mathrm{A}=\text { prost } \\
\text { argeted } \mathrm{t} \\
\mathrm{Hb}=\mathrm{Her} \\
\text { ase; NSE }\end{array}$ & $\begin{array}{l}\text { ex; TUR- } \\
\text { e-specifc } \\
\text { rapy; PSA } \\
\text { globin; A } \\
\text { eeuron-sf }\end{array}$ & $\begin{array}{l}\text { state = transu } \\
\text { mbrane antig } \\
\text { orostate- spec } \\
\text { alkaline phos } \\
\text { fic enolase; C }\end{array}$ & $\begin{array}{l}\text { hral } \\
\text { ADT = } \\
\text { atase; } \\
=\end{array}$ \\
\hline
\end{tabular}

At baseline, FT levels below the detection limit were more common in group 3 than in groups 1 and 2 ( $p<$ 0.001). PSA, FT and TT were higher in group 1 than in groups 2 and 3 , whereas hemoglobin was lower. 
Overall, $95.5 \%$ patients had bone metastases and $62.7 \%$ had soft tissue metastases. A median of two organs were involved by metastatic disease. All other characteristics were well-balanced (Table 1).

\section{Effects of docetaxel chemotherapy on TT and FT}

Serum levels of TT were reduced from a median of $0.12 \mathrm{ng} / \mathrm{mL}$ at baseline to $0.05 \mathrm{ng} / \mathrm{mL}$ at nadir $(p=$ 0.014 ) and FT from $0.32 \mathrm{pg} / \mathrm{mL}$ at baseline to non-detectable levels at nadir during docetaxel chemotherapy ( $p=0.006$; Fig. 1). 49/60 patients had TT levels under the detection limit during chemotherapy, but only $23 / 55$ at baseline (all receiving abiraterone) $(p=0.014)$ (Fig. 1$)$. Similarly, 46 of 61 patients had nadir FT levels below the detection limit in contrast to only 17/58 at baseline (who were treated with abiraterone at that time point) $(p=0.006)$ (Fig. 1; Table 2). FT suppression below the detection limit was significantly associated with PSA response $(p=0.008$; odds ratio $0.11195 \% \mathrm{Cl} 0.022-$ 0.564). While 6/7 (85.7\%) mCNPC patients achieved FT suppression to non-detectable levels, only $11 / 20$ (55\%) mCRPC patients and 8/27 (29.6\%) patients of the mCRPC-ART group had complete FT suppression $(p=0.23$ and $p=0.006$, respectively; Fig. $2 \mathrm{~A})$. The median time to $\mathrm{FT}$ nadir was 14 days $(95 \% \mathrm{Cl}$ $12.8 / 28.5, \mathrm{n}=37)$. 
Table 2

- Treatment outcome, survival and clinical responses

\begin{tabular}{|c|c|c|c|c|c|}
\hline & $\begin{array}{l}\text { All } \\
n=67\end{array}$ & $\begin{array}{l}\text { Group } 1 \\
\text { mCNPC } \\
n=7\end{array}$ & $\begin{array}{l}\text { Group } 2 \\
\text { mCRPC } \\
n=26\end{array}$ & $\begin{array}{l}\text { Group } 3 \\
\text { mCRPC- } \\
\text { ART } \\
\mathrm{n}=34\end{array}$ & $\begin{array}{l}p- \\
\text { value }\end{array}$ \\
\hline Initial dosage, no.(\%) & $45(67.2)$ & $6(85.7)$ & $24(92.3)$ & $15(44.1)$ & \\
\hline $75 \mathrm{mg} / \mathrm{m}^{2}$ & $15(22.4)$ & 0 & $1(3.9)$ & $14(41.2)$ & \\
\hline $50 \mathrm{mg} / \mathrm{m}^{2}$ & $7(10.5)$ & $1(14.3)$ & $1(3.9)$ & $5(14.7)$ & \\
\hline \multicolumn{6}{|l|}{$35 \mathrm{mg} / \mathrm{m}^{2}$} \\
\hline Dose reduction, no.(\%) & $12(17.9)$ & $1(14.3)$ & $5(19.2)$ & $6(17.7)$ & \\
\hline No. of cycles, median (range) & $8(2-108)$ & 17 & 12 & 6.5 & \\
\hline $\begin{array}{l}\text { Cumulative dose, median } \\
\text { (range) }\end{array}$ & $\begin{array}{l}663(100- \\
8100)\end{array}$ & 1275 & 750 & 450 & $<0.001$ \\
\hline $\begin{array}{l}\text { Duration of CTX in months, } \\
\text { median (range) }\end{array}$ & $7(1-78)$ & 15 & 10.5 & 6 & 0.004 \\
\hline OS in months $(95 \% \mathrm{Cl})$ & $\begin{array}{l}25.7(18.9- \\
32.5)\end{array}$ & $\begin{array}{l}33.7 \\
(30.9- \\
36.5)\end{array}$ & $\begin{array}{l}27.4 \\
(14.2- \\
40.6)\end{array}$ & $\begin{array}{l}16.8 \\
(16.2- \\
17.4)\end{array}$ & 0.002 \\
\hline PFS in months $(95 \% \mathrm{Cl})$ & $\begin{array}{l}7.8(3.9- \\
11.7)\end{array}$ & $\begin{array}{l}15.4 \\
(14.7- \\
16.2)\end{array}$ & $\begin{array}{l}11.3(6.3- \\
16.4)\end{array}$ & $\begin{array}{l}5.5(2.5- \\
8.6)\end{array}$ & 0.018 \\
\hline PSA response, no.(\%) & $43(64.2)$ & $7(100)$ & $19(73.1)$ & $17(50)$ & 0.014 \\
\hline $\begin{array}{l}\text { PSA reduction (\%), median } \\
\text { (range) }\end{array}$ & $\begin{array}{l}-66.5(+100- \\
(-99.9))\end{array}$ & -99.7 & -70.9 & -50 & 0.007 \\
\hline $\begin{array}{l}\text { Time to PSA response in days, } \\
\text { median (range) }\end{array}$ & $44(7-329)$ & 21 & 44 & 48 & 0.031 \\
\hline $\begin{array}{l}\text { FT median (pg/mL), median } \\
\text { (range) }\end{array}$ & $\begin{array}{l}0.18(0.18- \\
1.85)\end{array}$ & 0.18 & 0.21 & 0.18 & $<0.001$ \\
\hline $\begin{array}{l}\text { FT nadir (pg/mL), median } \\
\text { (range) }\end{array}$ & $0.18(0.18-1)$ & 0.18 & 0.18 & 0.18 & 0.001 \\
\hline $\begin{array}{l}\text { FT reduction (\%), median } \\
\text { (range) }\end{array}$ & $\begin{array}{l}-91.3(+33- \\
(-100))\end{array}$ & -100 & -100 & 0 & \\
\hline
\end{tabular}

CTX = Chemotherapy; $\mathrm{Cl}$ = confidence interval; OS = overall survival; $\mathrm{PFS}=$ progression - free survival; $\mathrm{PSA}=$ prostate-specific antigen; $\mathrm{FT}$ = free testosterone; $\mathrm{TT}$ = total testosterone; $\mathrm{NA}=$ not available; RECIST = response evaluation criteria in solid tumors; $P R=$ partial remission; $S D=$ stable disease; $P D$ = progressive disease; G-CSF = granulocyte-colony stimulating factor. 


\begin{tabular}{|c|c|c|c|c|c|}
\hline & $\begin{array}{l}\text { All } \\
n=67\end{array}$ & $\begin{array}{l}\text { Group } 1 \\
\text { mCNPC } \\
n=7\end{array}$ & $\begin{array}{l}\text { Group } 2 \\
\text { mCRPC } \\
n=26\end{array}$ & $\begin{array}{l}\text { Group } 3 \\
\text { mCRPC- } \\
\text { ART } \\
\mathrm{n}=34\end{array}$ & $\begin{array}{l}p- \\
\text { value }\end{array}$ \\
\hline $\begin{array}{l}\text { TT median }(\mathrm{ng} / \mathrm{mL}) \text {, median } \\
\text { (range) }\end{array}$ & $\begin{array}{l}0.08(0.05- \\
0.34)\end{array}$ & 0.05 & 0.1 & 0.05 & 0.03 \\
\hline $\begin{array}{l}\text { TT nadir ( } \mathrm{ng} / \mathrm{mL} \text { ), median } \\
\text { (range) }\end{array}$ & $\begin{array}{l}0.05(0.05- \\
0.35)\end{array}$ & 0.05 & 0.05 & 0.05 & \\
\hline $\begin{array}{l}\text { TT reduction (\%) median } \\
\text { (range) }\end{array}$ & $\begin{array}{l}-62.5(+120- \\
(-100))\end{array}$ & -100 & -43.8 & 0 & 0.043 \\
\hline \multirow{6}{*}{$\begin{array}{l}\text { Radiographic response (bone), } \\
\text { no.(\%) } \\
\text { improved } \\
\text { mixed } \\
\text { stable } \\
\text { progression }\end{array}$} & $n=64$ & $\mathrm{n}=7$ & $\mathrm{n}=25$ & $\mathrm{n}=32$ & \multirow[t]{6}{*}{0.034} \\
\hline & $10(15.6)$ & $3(42.9)$ & $2(8)$ & $5(16)$ & \\
\hline & $4(6.3)$ & 0 & $2(8)$ & $2(6.3)$ & \\
\hline & $29(45.3)$ & $4(57.1)$ & $12(48)$ & $13(40.6)$ & \\
\hline & $13(20.3)$ & 0 & $7(28)$ & $6(18.8)$ & \\
\hline & & & NA: 2 (8) & $\begin{array}{l}\text { NA: } 6 \\
(18.8)\end{array}$ & \\
\hline (soft tissue), no.(\%) & $\mathrm{n}=42$ & $\mathrm{n}=5$ & $\mathrm{n}=14$ & $n=23$ & 0.002 \\
\hline RECIST (\%), median & -28 & -75.8 & -21.9 & -10 & 0.017 \\
\hline PR & $18(42.9)$ & $5(100)$ & $5(35.7)$ & $8(34.8)$ & \\
\hline SD & $9(21.4)$ & 0 & $5(35.7)$ & $4(17.4)$ & \\
\hline \multirow[t]{2}{*}{ PD } & $11(26.2)$ & 0 & $3(21.4)$ & $8(34.8)$ & \\
\hline & NA: $6(2.6)$ & & NA: $1(7.1)$ & NA: $3(8.8)$ & \\
\hline Blood transfusion, no. (\%) & $22(32.8)$ & $2(28.6)$ & $7(26.9)$ & $13(38.2)$ & \\
\hline G-CSF, no. (\%) & $9(13.4)$ & $2(28.6)$ & $2(7.7)$ & $5(14.7)$ & \\
\hline
\end{tabular}

CTX = Chemotherapy; $\mathrm{Cl}$ = confidence interval; OS = overall survival; $\mathrm{PFS}=$ progression-free survival; $\mathrm{PSA}=$ prostate-specific antigen; $\mathrm{FT}$ = free testosterone; $\mathrm{TT}=$ total testosterone; $\mathrm{NA}$ = not available; RECIST = response evaluation criteria in solid tumors; $\mathrm{PR}=$ partial remission; $\mathrm{SD}=$ stable disease; $\mathrm{PD}$ = progressive disease; G-CSF = granulocyte-colony stimulating factor. 


\begin{tabular}{|c|c|c|c|c|c|}
\hline & $\begin{array}{l}\text { All } \\
n=67\end{array}$ & $\begin{array}{l}\text { Group } 1 \\
\text { mCNPC } \\
\mathrm{n}=7\end{array}$ & $\begin{array}{l}\text { Group } 2 \\
\text { mCRPC } \\
n=26\end{array}$ & $\begin{array}{l}\text { Group } 3 \\
\text { mCRPC- } \\
\text { ART } \\
n=34\end{array}$ & $\begin{array}{l}p- \\
\text { value }\end{array}$ \\
\hline Adverse events, no.(\%) & $47(70.2)$ & $6(85.7)$ & $17(65.4)$ & $24(70.6)$ & 0.017 \\
\hline Fatigue & $24(35.8)$ & 0 & $8(30.8)$ & $16(47.1)$ & 0.041 \\
\hline Dyspnea & $24(35.8)$ & $2(28.6)$ & $8(30.8)$ & $14(41.2)$ & 0.042 \\
\hline Nausea & $9(13.4)$ & 0 & $1(3.9)$ & $8(23.5)$ & 0.012 \\
\hline Diarrhea & 7 (10.5) & $1(14.3)$ & $3(11.5)$ & $3(8.8)$ & \\
\hline Constipation & $16(23.9)$ & $2(28.6)$ & $6(23.1)$ & $8(23.5)$ & \\
\hline Infection & $14(20.9)$ & 0 & $9(34.6)$ & $5(14.7)$ & \\
\hline PNP & $4(6)$ & 0 & $4(15.4)$ & 0 & \\
\hline \multicolumn{6}{|l|}{ Nail changes } \\
\hline \multirow{2}{*}{$\begin{array}{l}\text { Hemtatological toxicities, no. } \\
(\%)\end{array}$} & $49(73.1)$ & $7(100)$ & $20(76.9)$ & $22(64.7)$ & \\
\hline & \multicolumn{5}{|c|}{ Anemia grade $1+2$} \\
\hline \multicolumn{6}{|l|}{ Anemia grade $3+4$} \\
\hline Leukopenia grade $1+2$ & $27(40.3)$ & $3(42.9)$ & 10 (38.) & $14(41.2)$ & \\
\hline Leukopenia grade $3+4$ & $18(26.9)$ & $3(42.9)$ & $8(30.8)$ & $7(20.6)$ & \\
\hline Neutropenia grade $1+2$ & 11 (16.4) & 0 & $4(15.4)$ & 7 (20.6) & 0.047 \\
\hline Neutropenia grade $3+4$ & $22(32.8)$ & $4(57.1)$ & $11(42.3)$ & $7(20.6)$ & \\
\hline Thrombopenia grade $1+2$ & $22(32.8)$ & $4(57.1)$ & $9(34.6)$ & $9(26.5)$ & \\
\hline Thrombopenia grade $3+4$ & $3(4.5)$ & 0 & $2(7.7)$ & $1(2.9)$ & \\
\hline \multicolumn{6}{|c|}{$\begin{array}{l}\text { CTX = Chemotherapy; } \mathrm{Cl}=\text { confidence interval; } \mathrm{OS}=\text { overall survival; } \mathrm{PFS}=\text { progression-free survival; } \\
\mathrm{PSA}=\text { prostate-specific antigen; } \mathrm{FT}=\text { free testosterone; } \mathrm{TT}=\text { total testosterone; } \mathrm{NA}=\text { not available; } \\
\text { RECIST = response evaluation criteria in solid tumors; } \mathrm{PR}=\text { partial remission; } \mathrm{SD}=\text { stable disease; } \mathrm{PD} \\
\text { = progressive disease; G-CSF = granulocyte-colony stimulating factor. }\end{array}$} \\
\hline
\end{tabular}

\section{Clinical outcome}

Patients underwent a median of eight cycles of docetaxel (range 2-108) and were treated with a median cumulative dosage of $663 \mathrm{mg} / \mathrm{m}^{2}$ (range $100-8100 \mathrm{mg} / \mathrm{m}^{2}$ ). Group 1 (mCNPC) and group 2 (mCRPC) received a higher cumulative docetaxel dose than group 3 (mCRPC-ART) $\left(1,275 \mathrm{mg} / \mathrm{m}^{2} \mathrm{vs} .450 \mathrm{mg} / \mathrm{m}^{2}, p\right.$ $<0.001,750 \mathrm{mg} / \mathrm{m}^{2}$ vs. $450 \mathrm{mg} / \mathrm{m}^{2}, p=0.004$, respectively, Table 2$)$. The overall PSAR rate ( $\left.\geq 50 \%\right)$ was 43/67 (64.2\%): 7/7 (100\%) in group 1 (mCNPC), 19/26 (73.1\%) in group 2 (mCRPC) and 17/34 (50\%) in 
group 3 (mCRPC-ART) ( $p=0.13$ and $p=0.014$, respectively; Fig. 2B). The overall median PSAR was $66.5 \%$ (range +100 to $-99.9 \%$ ): a median of $-99.7 \%$ in group $1 ;-70.9 \%$ in group 2 and $-50 \%$ in group 3 , respectively $(p=0.007)$ (Table 2$)$. The median time to PSAR $(\geq 50 \%)$ was 44 days (range 7-329).

There was a higher rate of improvement of bone lesions in group 1 than group 2 (42.9\% vs. $7.7 \%, p=$ 0.034 , Table 2). The shrinkage of soft tissue metastases objectified by RECIST 1.1 was significantly higher in group 1 than group $2(-75.8 \%$ vs.-21.9, $p=0.002$; Fig. $2 \mathrm{C} \&$ Table 2$)$. The rate of partial remission was also significantly higher in group 1 than in groups 2 and $3(71.4 \%$ vs. $19.2 \%, p=0.017$ and $71.4 \%$ vs. $23.5 \%, p=0.02$, respectively).

Median OS was 25.7 months ( $95 \% \mathrm{Cl} 18.9-32.5)$ in all patients. mCNPC patients experienced a median OS of 33.7 months $(95 \% \mathrm{Cl} 30.9-36.5)$, whereas patients in group 3 (mCRPC-ART) had a median OS of 16.8 months $(95 \% \mathrm{Cl} 16.2-17.4 ; p=0.002)$. Median PFS in all patients was 7.8 months $(95 \% \mathrm{Cl} 3.9-$ 11.7): 15.4 months (95\% $\mathrm{Cl} 14.7-16.2)$ for group 1, 11.3 months $(95 \% \mathrm{Cl} 6.3-16.4)$ for group 2 and 5.5 months ( $95 \% \mathrm{Cl} 2.5-8.6)$ in group 3 ( $p=0.033$ and $p=0.018$, respectively; Table 2, Fig. 3 ).

\section{Multivariate analyses}

Univariate analyses of baseline parameters associated with OS are summarized in Table 3 . In multivariate analyses with stepwise regression, only prior ART and the presence of soft tissue metastases (lymphatic, hepatic, pulmonary, brain) remained independent predictors of a shorter OS (Table 3). 
Table 3

Baseline factors influencing OS and PFS: univariate and multivariate analysis

\begin{tabular}{|c|c|c|c|c|}
\hline & $\begin{array}{l}\text { Univariate } \\
\text { analysis } \\
\text { HR }(95 \% \mathrm{Cl})\end{array}$ & $p$ value & $\begin{array}{l}\text { Multivariate } \\
\text { analysis } \\
\text { HR }(95 \% \mathrm{Cl})\end{array}$ & $p$-value \\
\hline Overall survival & $2.71(1.37-5.34)$ & 0.004 & $2.56(1.28-5.11)$ & 0.008 \\
\hline$E C O G>1$ & $2.27(1.41-3.65)$ & 0.001 & $3.46(1.81-6.6)$ & $<0.001$ \\
\hline Group (1-3) & $3.09(1.69-5.67)$ & $<0.001$ & $0.28(0.14-0.57)$ & $<0.001$ \\
\hline Prior ART overall & $0.39(0.15-0.99)$ & 0.047 & & \\
\hline Response to ART & $2.8(1.45-5.41)$ & 0.002 & & \\
\hline Previous prednisone & $2.05(1.01-4.19)$ & 0.048 & & \\
\hline FT $<$ detection level at baseline & $1.93(1.01-3.68)$ & 0.047 & & \\
\hline CRP $>$ median & $3.46(1.35-8.88)$ & 0.01 & & \\
\hline NLR $>3$ & $1.73(1.29-2.31)$ & $<0.001$ & & \\
\hline Number of organs & $3.17(1.67-6)$ & $<0.001$ & & \\
\hline Soft tissue metastases & $2.38(1.33-4.43)$ & 0.003 & & \\
\hline Lymphatic metastasis & $3.05(1.43-6.48)$ & 0.004 & & \\
\hline Hepatic metastasis & $2.57(1.39-4.74)$ & 0.002 & & \\
\hline PD soft tissue and bone baseline & $2.1(1.16-3.8)$ & 0.015 & & \\
\hline Narcotics required & $0.31(0.16-0.62)$ & 0.001 & & \\
\hline $\begin{array}{l}\text { Treatment-dependent } \\
\text { parameters } \star \star\end{array}$ & $0.49(0.24-1.0)$ & 0.049 & & \\
\hline \multicolumn{5}{|l|}{ FT reduction $=100 \%$} \\
\hline FT median $<0.3 \mathrm{pg} / \mathrm{mL}$ & & & & \\
\hline
\end{tabular}

$\mathrm{HR}=$ hazard ratio; $\mathrm{Cl}=$ confidence interval; $\mathrm{ECOG}=$ Eastern cooperative oncology group; $\mathrm{ART}=$ androgen receptor targeted therapy; $\mathrm{FT}$ = free testosterone; $\mathrm{CRP}=\mathrm{C}$-reactive protein; NLR = neutrophile to lymphocyte ratio; $\mathrm{PD}=$ progressive disease; $\mathrm{PSA}$ = prostate-specific antigen; $\mathrm{FT}$ = free testosterone

$\star *=$ time-dependent covariate analysis revealed for $\mathrm{FT}$ reduction $=100 \%$ and $\mathrm{FT}$ median $<0.3 \mathrm{pg} / \mathrm{mL}$ $p>0.05$ for overall survival applying $T_{-}, \ln \left(T_{-}\right)$and $T \geq 365$ days

$\forall=\ln (T)$ revealed for PSA response $p=0.015$, for the remaining time dependent parameters using $T_{-}, \ln (T)$ and $T \geq 365$ days there was no significant time-dependence. 


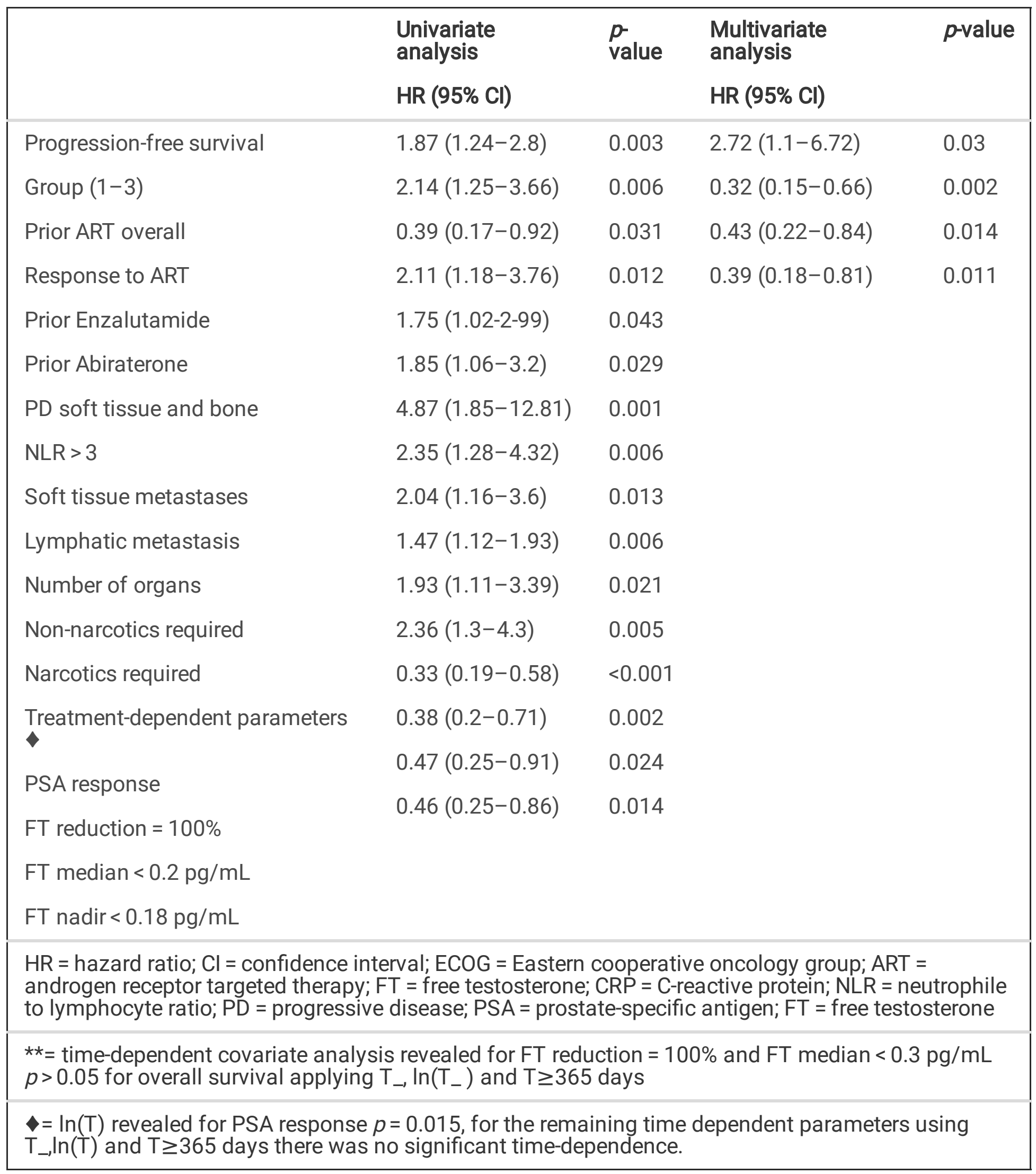

In univariate and time-dependent covariate analyses ( $T_{-} ; \operatorname{In}\left(T_{-}\right) ; T_{-} \geq 365$ days; data not shown) of treatment-dependent parameters (e.g. FT-, PSA-response), only FT median $<0.3 \mathrm{pg} / \mathrm{mL}$ and complete FT suppression (reduction of $100 \%$ ) during docetaxel therapy were associated with a longer OS. In 
multivariate analysis, complete FT reduction (100\%) remained an independent predictor for better OS (Table 3).

In addition to extended time-dependent Cox modelling, log (-log(survival probability)) and conditional landmark analyses were performed and demonstrated that the FT reduction $=100 \%$ during docetaxel therapy had no association with time regarding OS (data not shown).

In multivariate analysis of the baseline parameters, only the requirement of non-narcotics remained an independent predictor of a better PFS (Table 3). In univariate analysis of treatment-dependent parameters, PSAR, FT suppression (100\%), FT median values $<0.2 \mathrm{pg} / \mathrm{mL}$ and FT nadir values $<0.18$ $\mathrm{pg} / \mathrm{mL}$ during docetaxel therapy were associated with a better PFS. In multivariate analysis with stepwise regression, only PSAR, FT reduction $=100 \%$ and $\mathrm{FT}$ nadir values $<0.18 \mathrm{pg} / \mathrm{mL}$ remained independent predictors of a better PFS. Applying extended time-dependent Cox modelling ( $T_{-} ; \ln \left(T_{-}\right) ; T_{-} \geq 365$ days), $\log (-\log ($ survival probability)) and conditional landmark analyses demonstrated that these parameters had no association with time, with the exception of $\ln \left(T_{-}\right)$for PSAR, which revealed a significant timedependence ( $p=0.015$, Table 3 ) (data not shown).

\section{Discussion}

In this study, we demonstrate that TT and FT serum levels are reduced during docetaxel chemotherapy and that FT suppression under the detection limit (100\%) resulted in better PFS and OS in MCNPC and mCRPC patients, but not in mCRPC patients with a history of ART. Interestingly, in contrast to TT, only FT was a significant predictor for PFS and OS, demonstrating a major biological role of FT for treatment outcome. mCRPC-ART patients had a significantly lower FT reduction rate due to low FT levels at baseline (8/27 versus $6 / 7$ and $11 / 20$, respectively) and FT reduction was no longer a predictor for better PFS or OS (Table 2). mCRPC-ART patients experienced a lower PSAR and shorter PFS and OS. These results are consistent with several previous studies that showed decreased efficacy of docetaxel in PC patients with a history of ART $[11,12,21,22]$. Our data suggest that the worst clinical outcome of mCRPC-ART patients towards docetaxel is due to progressing castration resistance.

Multiple lines of evidence suggest that docetaxel and prednisone might directly interfere with testosterone biosynthesis and metabolism in $\mathrm{MPC}$ patients and contribute to this FT suppressing effect. Prednisone was demonstrated to lower serum TT, androstenedione, dehydroepiandrosterone (DHEA) and dehydroepiandrosterone sulfate (DHEAS) levels in some metastatic PC patients by suppressing the hypothalamic-pituitary-adrenal axis, but had no antitumor activity in $\mathrm{mCRPC}[23,24]$. Consistent with this observation, a history of previous prednisone treatment had no effect in multivariate analyses on PFS or OS in our study.

The role of docetaxel in reducing testosterone is less clear, although serum androgens (TT, androstendione and DHEA) decline during docetaxel treatment [5]. Docetaxel metabolism is largely catalyzed by CYP3A4 [25] and docetaxel was shown to induce CYP3A4, which is responsible for the greatest portion of testosterone $6 \beta$ - and 16ß-hydroxylation [26-28]. CYP3A4 induction may lower 
testosterone levels by inactivation through $6 \beta$ - and $16 \beta$-hydroxylation. The effect of docetaxel on CYP17A1 is unclear [26-28].

Franke et al. reported castration-dependent pharmacokinetics of docetaxel in PC patients. Docetaxel clearance was increased by approximately $100 \%$ in castrated men and was associated with a two-fold reduction in area under the curve, although hepatic activity of CYP3A4 was unchanged [29]. Conversely, castration-naïve patients were exposed to higher amounts of the drug, accompanied by more severe hematotoxicity [29]. This study also demonstrated that lower intracellular docetaxel levels caused by lower levels of testosterone resulted in a lower response rate to treatment [29]. Consistent with these results, group 1 patients (mCNPC) had a significantly higher rate of grade 3 and 4 neutropenia compared to group 3 patients (mCRPC-ART) in our study ( $57.1 \%$ vs. $20.6 \%, p=0.047$; Table 2$)$ and a significantly better clinical outcome (Figs. $2 \& 3$, Table 2).

Ryan et al. showed that conversion from higher to lower androgen levels (e.g. above/below median) during docetaxel therapy contributed to superior survival as the reduction is the driving mechanism behind the clinical responses [5]. Consistent with these findings, 6/7 (85.7\%) mCNPC patients in our study underwent a complete $(=100 \%)$ and one patient a nearly complete $(99.3 \%)$ FT reduction and had a PSA response rate of $100 \%$.

In recent years, several large phase 3 trials in patients with mCNPC (e.g. CHAARTED, STAMPEDE, GETUG3, LATTITUDE, TITAN, PREVAIL) demonstrated that the addition of docetaxel and ART (abiraterone, apalutamide and enzalutamide) to ADT is associated with significant improvements in PFS and/or OS compared to ADT alone [30]. Our data demonstrate that docetaxel therapy is associated with similarly low testosterone levels $(\mathrm{FT}+\mathrm{TT}$ ) as achieved by Abiraterone + ADT (Fig. 1, Table 1\&2).

Our study has some limitations, due to its retrospective design. The population size is small and FT and TT measurements - although excessive in number - were not always assessed on a regular basis (e.g. weekly). Furthermore, progression was mainly due to PSA progression (e.g. PSA progression or radiographic progression, whichever presented first). Despite all of our efforts to address possible leadtime bias (extended time-dependent Cox modelling ( $T_{-} ; \ln \left(T_{-}\right) ; T_{-} \geq 365$ days), $\log$ (-log(survival probability)) and conditional landmark analyses), there is still the risk that our analyses are subject to lead-time bias, as group 3 patients (mCRPC-ART) had more advanced disease at baseline compared to groups 1 (mCNPC) and 2 (mCRPC). Group 1 was small due to a recent trend towards abiraterone treatment in this setting and patients had a very high-volume disease that required intensive treatment. In addition, scanning intervals were not always uniformly assessed and confirmatory scans were not conducted in general.

\section{Conclusions}

This study represents the strongest evidence to date that FT plays a fundamental role during docetaxel chemotherapy. In mCNPC and mCRPC patients, complete FT suppression $(=100 \%)$ during chemotherapy was an independent predictor of PSAR, RR, PFS and OS. However, in mCRPC patients with a history of 
ART, FT was not linked to the clinical outcome. Our data suggest that castration-dependent pharmacokinetics of docetaxel seem to reduce its clinical effectiveness in mCRPC-ART patients.

\section{Declarations}

Declaration of interest: none.

Availability of data and materials: Supplementary appendix

Funding: This is an academic study. This work was sponsored and supported by Hannover Medical School $(\mathrm{MHH})$. The sponsor $(\mathrm{MHH})$ had no role in study design, in the collection, analysis and interpretation of data. Chemotherapy was provided as standard of care.

Authorship: PK, MAM, PI, SJB and CWMR helped write the manuscript. PK and CWMR designed the study.

AG supervised this research project.

Acknowledgments: We thank the patients and their families who participated in the study.

\section{References}

1. Crawford, E.D. et al. Androgen-targeted therapy in men with prostate cancer: evolving practice and future considerations. Prostate Cancer Prostatic Dis.22, 24-38 (2019)

2. Teo, M.Y., Rathkopf, D.E. \& Kantoff, P. Treatment of Advanced Prostate Cancer. Annu Rev Med.70, 479-499 (2019)

3. Sartor, O., de Bono, J.S. Metastatic Prostate Cancer. N England J Med.378, 645-657 (2018)

4. Scher, H.I. et al. Design and end points of clinical trials for patients with progressive prostate cancer and castrate levels of testosterone: recommendations of the Prostate Cancer Clinical Trials Working Group. J Clin Oncol. 26, 1148-1159 (2008)

5. Ryan, C.J. et al. Androgen decline and survival during docetaxel therapy in metastatic castration resistant prostate cancer (mCRPC). Prostate Cancer Prostatic Dis.23, 66-73 (2020)

6. Ryan, C.J. et al. Serum androgens as prognostic biomarkers in castration-resistant prostate cancer: results from an analysis of a randomized phase III trial. J Clin Oncol.31, 2791-2798 (2013)

7. Diver, M. Laboratory measurement of testosterone. Front Horm Res37, 21-31 (2009)

8. Morote, J. et al. Behavior of free testosterone in patients with prostate cancer on androgen deprivation therapy. Int J Biol Markers. Int J Biol Markers.20, 119-222 (2005)

9. Rove, K.O. et al. Maximal testosterone suppression in prostate cancer-free vs total testosterone. $\mathbf{8 3}$, 1217-1222 (2014)

10. von Klot, C.A. et al. Role of free testosterone levels in patients with metastatic castration-resistant prostate cancer receiving second-line therapy. Oncol Lett.13, 22-28 (2017) 
11. Delanoy, N. et al. Sequencing of Taxanes and New Androgen-targeted Therapies in Metastatic Castration-resistant Prostate Cancer: Results of the International Multicentre Retrospective CATS Database. Eur Urol Oncol.1, 467-475 (2018)

12. Mezynski, J. et al. Antitumour activity of docetaxel following treatment with the CYP17A1 inhibitor abiraterone: clinical evidence for cross-resistance? Ann Oncol.23, 2943-2947 (2012)

13. Lolli, C. et al. Testosterone levels and androgen receptor copy number variations in castrationresistant prostate cancer treated with abiraterone or enzalutamide. The Prostate.79, 1211-1220 (2019)

14. Gan, L. et al. Inhibition of the androgen receptor as a novel mechanism of taxol chemotherapy in prostate cancer. Cancer Res.69, 8386-8394 (2009)

15. Montgomery, R.B. et al. Maintenance of intratumoral androgens in metastatic prostate cancer: a mechanism for castration-resistant tumor growth. Cancer Res.68, 4447-4454 (2008)

16. Halabi, S. et al. Updated prognostic model for predicting overall survival in first-line chemotherapy for patients with metastatic castration-resistant prostate cancer. J Clin Oncol.32, 671-677 (2014)

17. Scher, H.I. et al. Trial Design and Objectives for Castration-Resistant Prostate Cancer: Updated Recommendations From the Prostate Cancer Clinical Trials Working Group 3. J Clin Oncol.34, 14021418 (2016)

18. Eisenhauer, E.A. et al. New response evaluation criteria in solid tumours: revised RECIST guideline (version 1.1). Eur J Cancer45, 228-247 (1990)

19. Bellera, C.A. et al. Variables with time-varying effects and the Cox model: some statistical concepts illustrated with a prognostic factor study in breast cancer. BMC Med Res Methodol.10, 20 (2010)

20. Giobbie-Hurder, A., Gelber, R.D. \& Regan, M.M. Challenges of guarantee-time bias. J Clin Oncol.31, 2963-2969 (2013)

21. de Bono, J.S. et al. Subsequent Chemotherapy and Treatment Patterns After Abiraterone Acetate in Patients with Metastatic Castration-resistant Prostate Cancer: Post Hoc Analysis of COU-AA-302. Eur Urol.71, 656-664 (2017)

22. Schweizer, M.T. et al. The influence of prior abiraterone treatment on the clinical activity of docetaxel in men with metastatic castration-resistant prostate cancer. Eur Urol. 66, 646-652 (2014)

23. Tannock, l. et al. Treatment of metastatic prostatic cancer with low-dose prednisone: evaluation of pain and quality of life as pragmatic indices of response. J Clin Oncol.7, 590-597 (1989)

24. Amato, R.J., Ellerhorst, J., Finn, L. \& Logothetis, C.J. Absence of antitumor activity with prednisone in patients with progressive androgen-independent prostate carcinoma. Urol Oncol.2, 70-73 (1996)

25. Marre, F. et al. Hepatic biotransformation of docetaxel (Taxotere) in vitro: involvement of the CYP3A subfamily in humans. Cancer Res.56, 1296-1302 (1996)

26. Kawano, S., Kamataki, T., Yasumori, T., Yamazoe, Y. \& Kato, R. Purification of human liver cytochrome P-450 catalyzing testosterone 6 beta-hydroxylation. J Biochem (Tokyo). 102, 493-501 (1987) 
27. Yamazaki, H. \& Shimada, T. Progesterone and testosterone hydroxylation by cytochromes P450 2C19, 2C9, and 3A4 in human liver microsomes. Arch Biochem Biophys.346, 161-169 (1997)

28. Nallani, S.C., Goodwin, B., Buckley, A.R., Buckley, D.J. \& Desai, P.B. Differences in the induction of cytochrome P450 3A4 by taxane anticancer drugs, docetaxel and paclitaxel, assessed employing primary human hepatocytes. Cancer Chemother Pharmacol. 54, 219-229 (2004)

29. Franke, R.M., Carducci, M.A., Rudek, M.A., Baker, S.D. \& Sparreboom, A. Castration-dependent pharmacokinetics of docetaxel in patients with prostate cancer. J Clin Oncol.28, 4562-4567 (2010)

30. Gillessen, S.et al. Management of Patients with Advanced Prostate Cancer: Report of the Advanced Prostate Cancer Consensus Conference 2019. Eur Urol.77, 508-547 (2020)

\section{Figures}
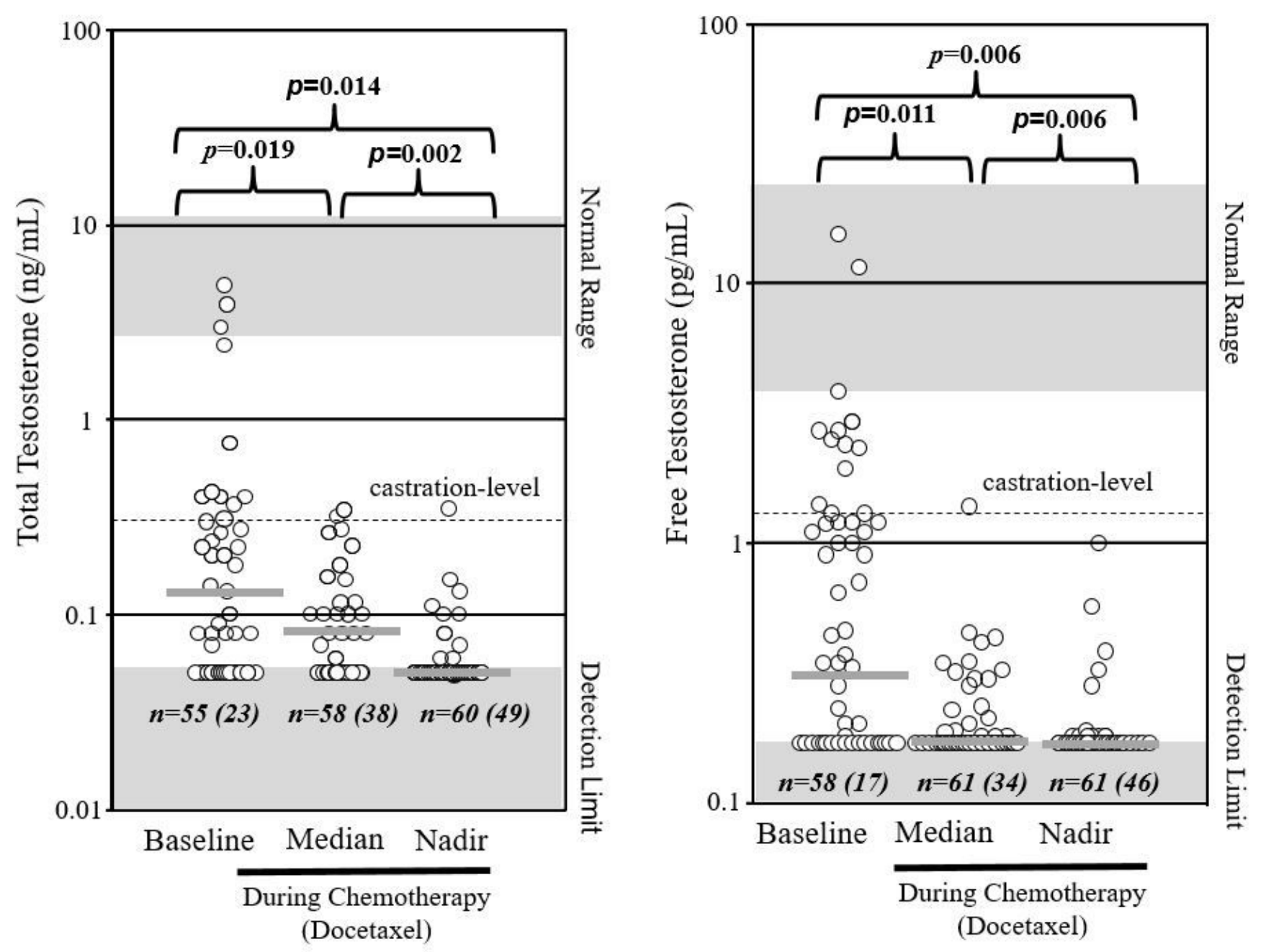

Figure 1

Distribution of baseline, median and nadir values of TT and FT levels. Castration level for TT is defined as $0.5 \mathrm{ng} / \mathrm{mL}$ [4] and for FT as $1.7 \mathrm{pg} / \mathrm{mL}$ [9]. Numbers in brackets demonstrate number of patients with 

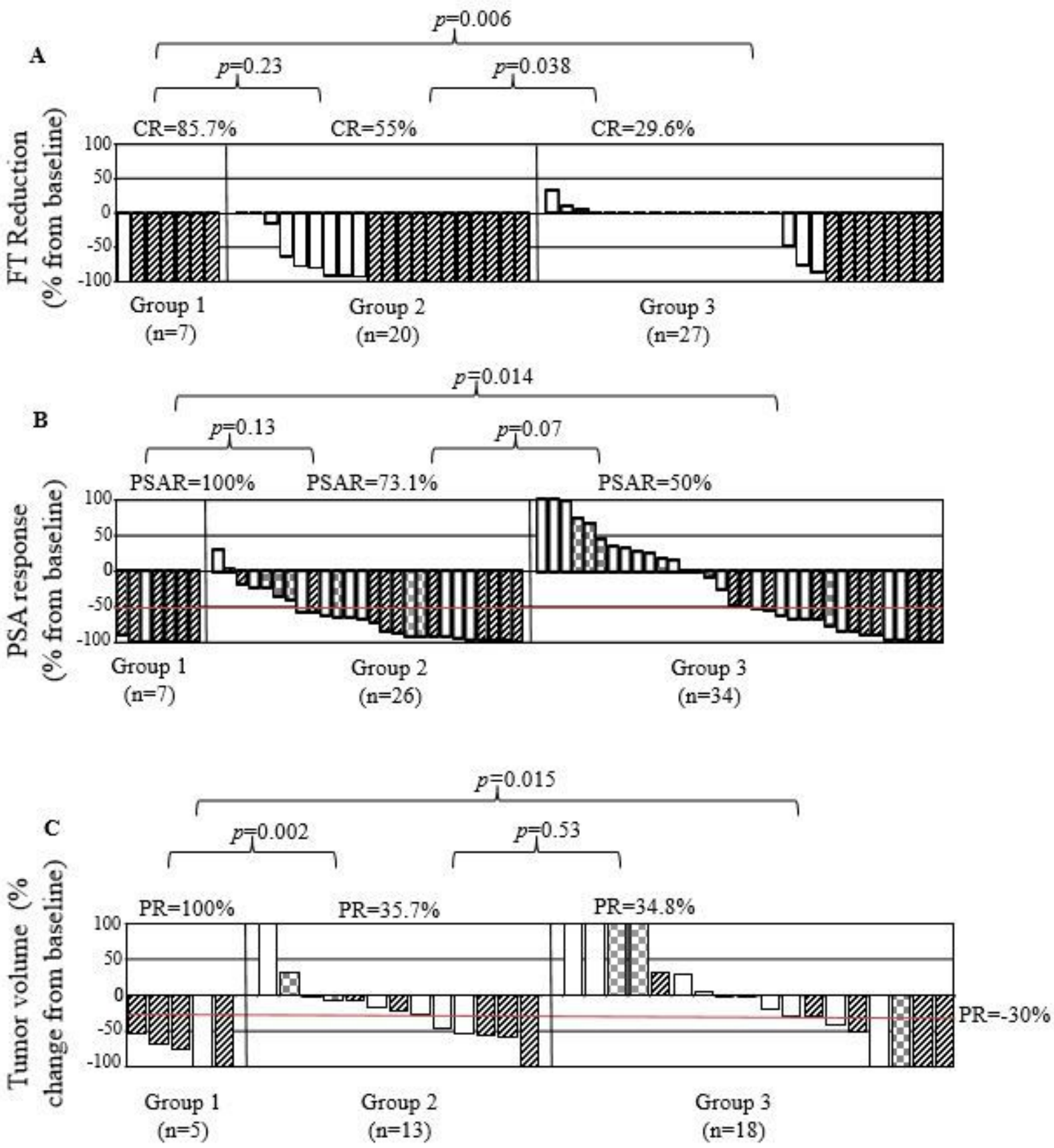

\section{Figure 2}

Response to docetaxel. A: FT reduction, complete reduction (CR) is marked striped in every waterfall plot. White columns show data of patients with no $\mathrm{CR}$ and squared columns show missing values. B: PSA response (PSAR) defined as decrease of $50 \%$ from baseline according to PCWG $2 / 3$ criteria $[4,17]$ during therapy is shown in the second plot. C: Shrinkage of soft tissue tumor metastases is given as percent from baseline according to RECIST 1.1 (response evaluation criteria in solid tumors) [18]. 

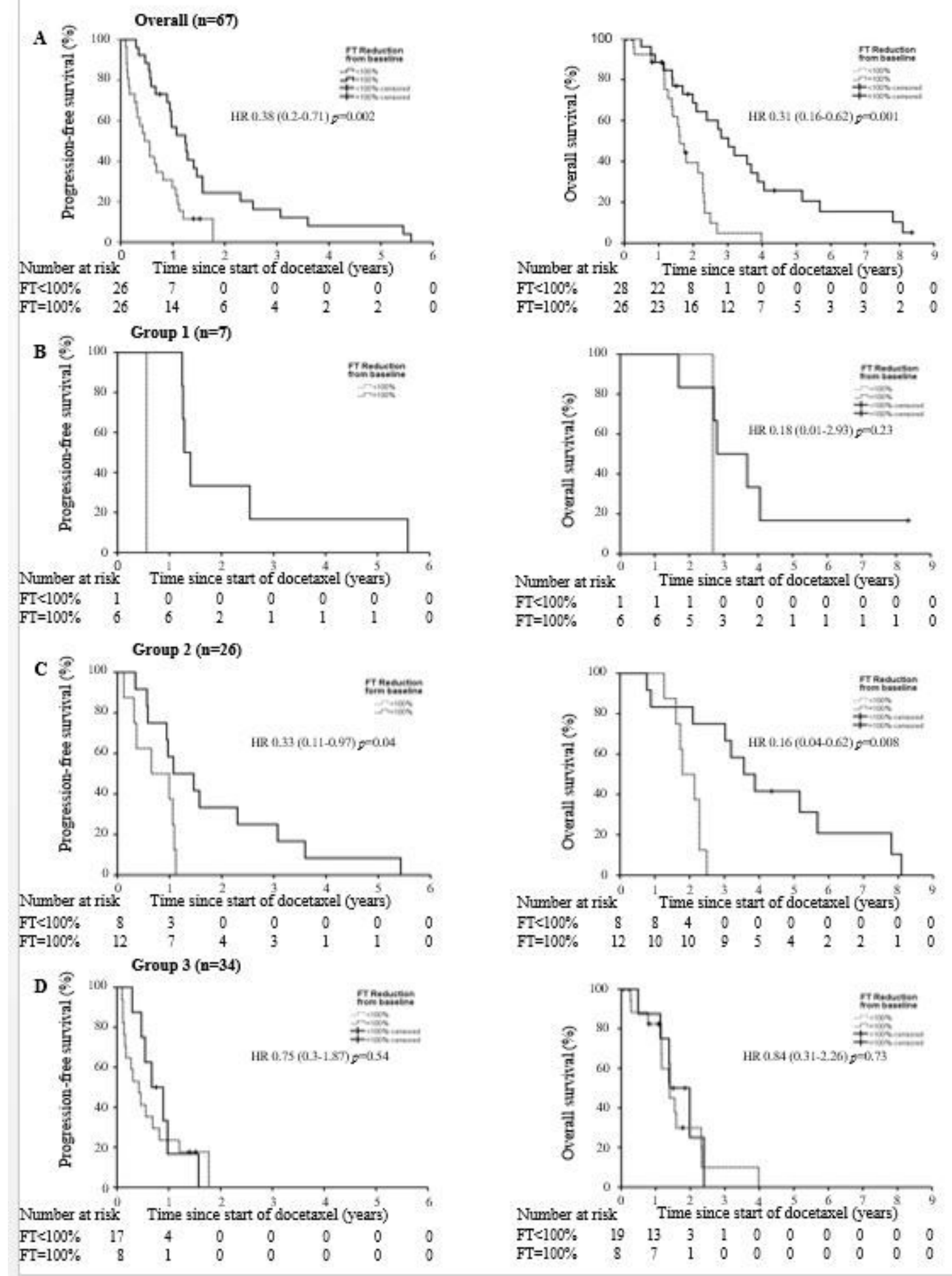

\section{Figure 3}

Kaplan-Meier curves for progression-free survival and overall survival for patients with FT reduction of $100 \%$ (black line) and for patients with $<100 \%$ reduction of FT (dotted line). 